\title{
On the impact of nuclear data uncertainties on LWR neutron dosimetry assessments
}

\author{
Alexander Vasiliev*, Marco Pecchia, Dimitri Rochman, Hakim Ferroukhi, and Erwin Alhassan \\ ${ }^{1}$ Laboratory for Reactor Physics and Thermal-Hydraulics (LRT), \\ Paul Scherrer Institute (PSI), Forschungsstrasse 111, 5232 Villigen, Switzerland
}

\begin{abstract}
In this work, an overview on the relevance of the nuclear data (ND) uncertainties with respect to the Light Water Reactors (LWR) neutron dosimetry is presented. The paper summarizes results of several studies realized at the LRT laboratory of the Paul Scherrer Institute over the past decade. The studies were done using the base LRT calculation methodology for dosimetry assessments, which involves the neutron source distribution representation, obtained based on validated CASMO/SIMULATE core follow calculation models, and the subsequent neutron transport simulations with the MCNP® software. The methodology was validated using as reference data results of numerous measurement programs fulfilled at Swiss NPPs. Namely, the following experimental programs are considered in the given overview: PWR "gradient probes" and BWR fast neutron fluence (FNF) monitors post irradiation examination (PIE). For the both cases, assessments of the nuclear data related uncertainties were performed. When appropriate, a cross-verification of the deterministic and stochastic based uncertainty propagation techniques is provided. Furthermore, the observations on which particular neutron induced reactions contribute dominantly to the overall ND-related uncertainties are demonstrated. The presented results should help with assessing the overall impact of the various nuclear data uncertainties with respect to dosimetry applications and provide relevant feedback to the nuclear data evaluators.
\end{abstract}

\section{Introduction}

The Paul Scherrer Institute (PSI) is involved in the reactor dosimetry assessments for Swiss nuclear power plants, which includes development and validation of calculation tools for the fast neutron fluence (FNF) predictions. The reference PSI FNF modelling methodology is based on the CASMO/SIMUATE/MCNP $®$ calculation route [1] (for details on the Monte Carlo NParticle $\AA$ MCNP $\AA$ Software, see https://mcnp.lanl.gov/; the calculations discussed in the given work were realised with MCNPX-2.4.0 and MCNPX-2.7.0 code versions). The methodology is oriented on hi-fidelity best-estimate plus uncertainty (BEPU) simulations relying on validated CASMO/SIMULATE core-follow models [2]. Results of different validation studies performed for both PWR and BWR type reactors have been already reported in a number of publications [3], [4], [5], [6]. Obviously, the BEPU methodological approach and associated validation studies require obtaining the calculated $(\mathrm{C})$ results accompanied with related uncertainty quantifications (UQ). Naturally, there are many contributors to the overall uncertainties of the $\mathrm{C} / \mathrm{E}$ results in the dosimetry applications. The nuclear data related part is obviously one of the most significant components, but not a solo one. It is therefore important to assess its typical values against other uncertainty components, to understand which type of input data is actually limiting further $\mathrm{C} / \mathrm{E}$ improvement.

*e-mail: alexander.vasiliev@psi.ch

\section{PWR dosimetry validation case}

The PWR dosimetry validation case considered in this work was presented in detail in [5]. Fig. 1 schematically illustrates locations where the "Gradient Probes" (GP) and "Scraping tests" (ST) irradiated samples were extracted from.

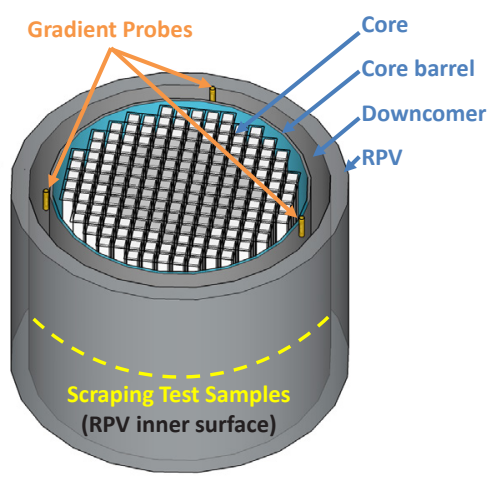

Figure 1. Simplified illustration on the Swiss PWR validation case.

The dosimetry information was derived based on two reactions: ${ }^{54} \mathrm{Fe}(\mathrm{n}, \mathrm{p}){ }^{54} \mathrm{Mn}$ and ${ }^{93} \mathrm{Nb}\left(\mathrm{n}, \mathrm{n}^{\prime}\right)^{93 m} \mathrm{Nb}$. For the present work, the analysis of the GP experimental data was selected for detailed discussion. 


\section{BWR dosimetry validation case}

The BWR dosimetry validation study considered in this work is similar to those reported in [3], [4]. The difference is that for the case analysed in this work, the experimental data corresponds to the FNF monitors extracted from the first surveillance capsules set, irradiated at the same BWR within the first 11 cycles of operation. Fig. 2 illustrates positions of the surveillance capsules (assuming the quarter core symmetry). The same dosimetry reactions were analysed as in the PWR case, plus ${ }^{63} \mathrm{Cu}(\mathrm{n}, \alpha){ }^{60} \mathrm{Co}$. Axially the FNF monitors were located close to the core mid-plane.

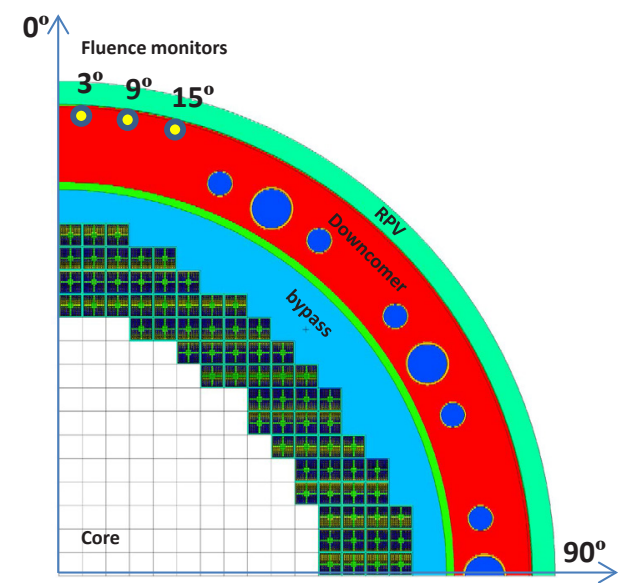

Figure 2. Simplified illustration on the Swiss BWR validation case.

Fig. 2 indicates that the central part of the core can be truncated in the MCNP model, since it does not contribute anyhow significantly to the dosimeters irradiation. Additional details can be found in $[3,4,6]$.

\section{Employed methodologies for ND UQ}

Two types of methods for ND UQ were employed in the presented work. The first one is the deterministic based approach which relies on the first-order sensitivity coefficients (SC) computed using MCNP differential operator capabilities (with the 'PERT' card). In this way, the ND UQ can be done using the well-known "sandwich rule" by folding the SC with covariance matrices (CM) obtained based on the information provided in the ND library.

The SC and CM were processed in the SCALE 44 energy group structure (in fact, only 11 energy bins of fast neutrons $(\mathrm{E}>1 \mathrm{MeV})$ were of relevance for the given study). ENDF/B-VII.1 CM files [7] were applied for ND UQ assessment. No uncertainties for the neutron multiplicity $\bar{v}$ and the fission neutron spectra $\chi$ were propagated in the deterministic approach.

The second method is based on stochastic sampling of the underlying ND and for that the PSI tool NUSS [8], [9], [10] was employed to randomly sample the ACE formatted ND files for subsequent MCNP sample calculations. In this case a fine 187 energy bin structure was applied for the same reactions as in the case of the deterministic -based approach, however, the CM for $\overline{v_{p}}$ and $\chi_{p}$ were added to the list of the perturbed ND. The same ENDF/B-VII.1 library [7] was used as the source of the required $\mathrm{CM}$ files.

\section{PWR case results}

The $\mathrm{C} / \mathrm{E}$ results obtained with the analysis of the PWR validation case were reported in [5]. In addition to that, a study on the impact of the ND library choice on the $\mathrm{C} / \mathrm{E}$ values can be found in [11]. In general, the obtained $\mathrm{C} / \mathrm{E}-1$ results lay within $\sim \pm 15 \%$. The differences in the FNF results due to the neutron transport ND libraries were found within $\sim 5 \%$. No information on the reference experimental data uncertainties was available at LRT.

Now, it is important to compare the above values with the associated ND UQ results. That is, for the case of the "gradient probe", the uncertainties calculated for ${ }^{54} \mathrm{Mn}$ and ${ }^{93 m} \mathrm{Nb}$ activities (obtained with the Sandwich rule, based on ENDF/B-VII.1 CM files and considering only neutron transport related cross-sections) were in the both cases about $5.5 \%$ [12].

The calculations with random ACE files were outlined in [10]. There, the uncertainty due to the neutron transport related ND (including $\overline{\nu_{p}}$ and $\left.\chi_{p}\right)$ on the FNF $(\mathrm{E}>1 \mathrm{MeV})$ results at the PWR pressure vessel inner surface around the core mid-height elevation were estimated as $7.6 \%$.

Ranking of the main uncertainties contributors in neutron transport modelling was also done [12], as illustrated in Fig. 3. Here, only the reactions having an impact on the calculation uncertainty above $0.5 \%$ are illustrated. The Monte Carlo related uncertainties are neglected as being negligible for the reported results. The labels show the reactor region, isotope and reaction MT number. Since the GP irradiation channels were attached to the core barrel, the downcomer (DC) coolant does not contribute to the calculation uncertainties, however this would not be the case for the ST data analysis.

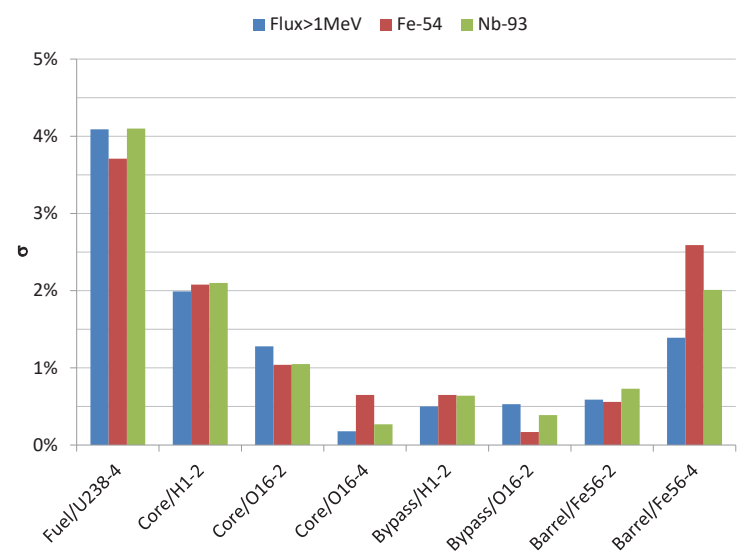

Figure 3. Main uncertainty contributors for the PWR GP dosimetry case.

The uncertainties only due to the dosimetry reactions were estimated as $2.2 \%$ for the ${ }^{54} \mathrm{Mn}$ activity and $4.4 \%$ for 
${ }^{93 m} \mathrm{Nb}$ activity, based on the ENDF/B-VII.1 CM data [12]. The effects of the dosimetry reaction cross-section libraries were also assessed, as shown in Fig. 4. In this case the ENDF/B-VII.1 library is used for the neutron transport simulations and the dosimetry cross-sections were taken either from ENDF/B-VII.1, JEFF-3.2 or from TENDL2013. The shown uncertainty bars correspond to the MCNP statistical errors only.
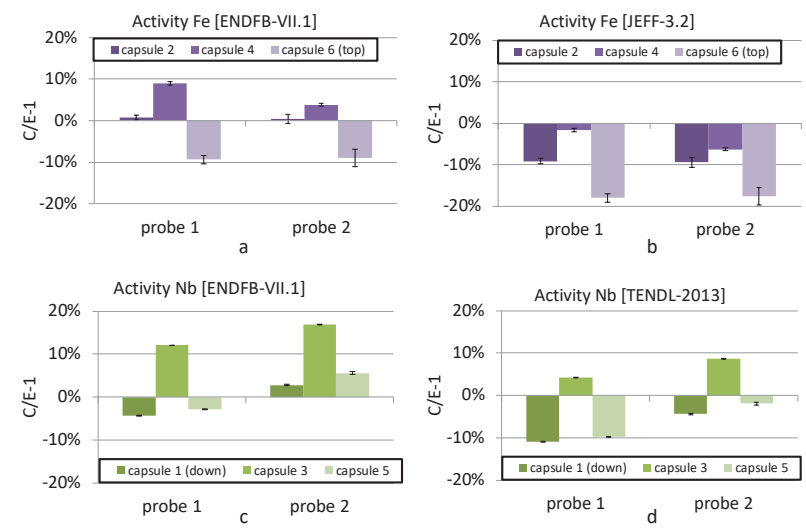

Figure 4. Impact of the dosimetry cross-sections on the PWR $\mathrm{C} / \mathrm{E}$ results.

One can notice that the impact of the library used for the dosimetry reactions can exceed the dosimetry ND uncertainties: $\sim 10 \%$ (see graphs 'a' and 'b' for ${ }^{54} \mathrm{Fe}$ ) vs. $\sim 2 \%$ (at $1 \sigma$-level), as reported above.

\section{BWR case results}

Some of the BWR validation studies were already reported in [3], [4]. However, the most recent results obtained in work [6] were not published yet, apart from the preliminary results [13]. Therefore, a representative illustration on the latest $\mathrm{C} / \mathrm{E}$ values on the dosimeter specific activities is given here on Fig 5.

The provided uncertainty bars include the contributions from the experimental data and the cross-section related uncertainties, together with the MCNP statistical uncertainties. The effect of the nuclear data library choice is also visible from Fig. 5: similar to the PWR case it is within $\sim 5 \%$. The ${ }^{54} \mathrm{Fe}(\mathrm{n}, \mathrm{p})$ and ${ }^{63} \mathrm{Cu}(\mathrm{n}, \alpha)$ cross-sections were taken from the same ND library as used for the neutron transport. The ${ }^{93} \mathrm{Nb}\left(\mathrm{n}, \mathrm{n}^{\prime}\right)^{93 m} \mathrm{Nb}$ reaction data was taken from the IRDFF-1.05 library.

One can see that typically the C/E-1 results are found within $\pm 20 \%$. Average $<\mathrm{C} / \mathrm{E}-1>$ biases for individual dosimeters can be reported on example of ENDF/B-VII.1 library results: $-9 \%,-1 \%$ and $-11 \%$ for ${ }^{93} \mathrm{Nb},{ }^{54} \mathrm{Fe}$ and ${ }^{63} \mathrm{Cu}$ respectively. Like for the case of the PWR model, the main ND uncertainties contributors in neutron transport modelling were also assessed, as illustrated in Fig. 6.

The overall neutron transport related ND uncertainty effects are about $12 \%^{1}$ on average for the both dosime-

\footnotetext{
${ }^{1}$ note that the effects from different reactions shall be summed up as variances, while the effects from the same reactions but from different reactor zones shall be summed up linearly
}

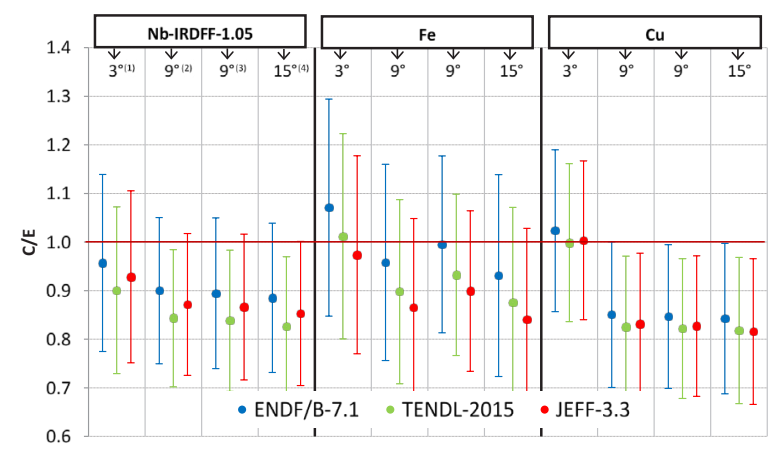

Figure 5. C/E values of the BWR dosimeter specific activities.

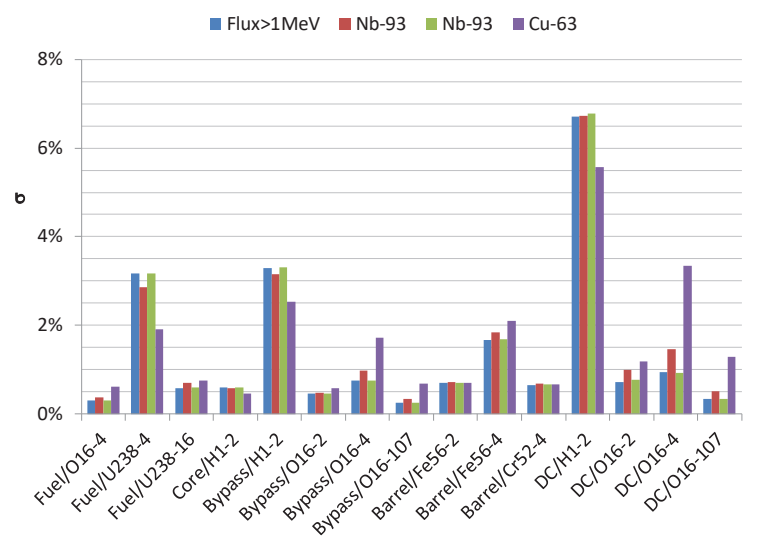

Figure 6. Main uncertainty contributors for the BWR RPV dosimetry case.

try monitors as well as for FNF. The major contribution corresponds to the elastic scattering on hydrogen in the DC region. For comparison, the uncertainty in FNF assessed with NUSS for the similar locations was reported in [10] as $14.5 \%$, noting that in the NUSS assessments $\overline{v_{p}}$ and $\chi_{p}$ uncertainties were added as compared to the 'sandwich rule' results.

\section{Discussion and Conclusions}

Table 1 summarizes in a rounded manner the results described above and obtained in the previous studies for the considered PWR and BWR reactors. 'PWR-GP' and 'PWR-ST' stand for the PWR dosimetry data obtained from the gradient probes and scraping test measurements. 'BWR-D' stands for the BWR dosimetry studies. The values marked with an asterisk were borrowed from [10] (obtained using ENDF/B-VII and for an extended set of the experimental data for the BWR-D case). For the 'BWRD' case, the newest values obtained in [13] and outlined here in Section 6 are added in parentheses. These values can also be compared with another $\mathrm{C} / \mathrm{E}$ data set reported in [3]. Deriving overall average $\mathrm{C} / \mathrm{E}$ results may require a special care (weighting procedure) and goes beyond the scope of the given paper. Nevertheless, one can notice that the ND related uncertainties are in general comparable to the $<\mathrm{C} / \mathrm{E}-1>$ variations. 
For the considered dosimetry applications and based on ENDF/B-VII.1 CM, the main uncertainty contributors are: ${ }^{238} \mathrm{U}\left(\mathrm{n}, \mathrm{n}^{\prime}\right) ;{ }^{1} \mathrm{H}(\mathrm{n}, \mathrm{n}) ;{ }^{16} \mathrm{O}(\mathrm{n}, \mathrm{n}),{ }^{16} \mathrm{O}\left(\mathrm{n}, \mathrm{n}^{\prime}\right) ;{ }^{56} \mathrm{Fe}(\mathrm{n}, \mathrm{n})$, ${ }^{56} \mathrm{Fe}\left(\mathrm{n}, \mathrm{n}^{\prime}\right)$. It also can be highlighted that the dosimetry relevant ${ }^{54} \mathrm{Fe}(\mathrm{n}, \mathrm{p}){ }^{54} \mathrm{Mn}$ and ${ }^{93} \mathrm{Nb}\left(\mathrm{n}, \mathrm{n}^{\prime}\right){ }^{93 m} \mathrm{Nb}$ reactions can noticeably differ between the modern ND libraries.

Table 1. Summary on the obtained C/E and UQ results.

\begin{tabular}{|c|c|c|c|}
\hline Model & PWR-GP & PWR-ST & BWR-D \\
\hline $\begin{array}{c}\sigma^{F N F},(\%) / \\
\text { "Sandwich rule" }\end{array}$ & $\begin{array}{c}5 \\
(E>0.1 \mathrm{MeV})\end{array}$ & NA & 12 \\
\hline $\begin{array}{c}\sigma^{\text {FNF, }}(\%) / \text { NUSS } \\
\text { (+nubar, +chi) }\end{array}$ & $8^{*}$ & $11^{*}$ & $15^{*}$ \\
\hline $\operatorname{MAX}(|C / E-1|),(\%)$ & 15 & 15 & $20(15)$ \\
\hline$\langle\mathrm{C} / \mathrm{E}-1\rangle, \mathrm{Fe}-54,(\%)$ & $5^{*}$ & $5^{*}$ & $18 *(-1)$ \\
\hline$<C / E-1>, N b-93,(\%)$ & $-1^{*}$ & $3^{*}$ & $9 *(-9)$ \\
\hline
\end{tabular}

Now, it is important to assess the magnitude of the NDrelated uncertainties in the typical dosimetry applications as compared with other sources of uncertainties and simulation biases. Obviously, one of the important sources of the uncertainties is the material compositions for the MCNP models. In the context of reactor dosimetry, however, of primary importance is actually the coolant density in the reactor core, bypass and downcomer. An influence of the coolant density distributions was considered, e.g., in [14] and it was found that refinement in the core bypass coolant density based on accurate accounting for the coolant density profiles vs. the previous rough estimation changed the results for the gradient probes reaction rates by as much as $\sim 10 \%$.

Another obvious source of the simulation uncertainties is the neutron source distribution, which is defined in the MCNP model, according to the PSE methodology, based on CASMO/SIMULATE thermal power and fuel compositions distributions. It is well known and it has also been demonstrated in PSI studies for Swiss reactors [1, 15], that with respect to the LWR ex-core dosimetry applications, the core peripheral fuel assemblies play the most important role since the neutrons generated in the inner parts of the core have low chances to reach the ex-core locations of interest (e.g., core barrel, RPV, etc.) still having the energy above $1 \mathrm{MeV}$ (which is the typical threshold for LWR dosimetry applications). It is also logical to expect, and this was actually demonstrated too in the LRT in-house validation studies [16], that the calculation accuracy of the reaction rates within the core is deteriorating at the peripheral fuel assemblies/axial nodes. This can be explained by the difficulty of catching the correct flux gradients and spectral changes at the interfaces between the reactor core and reflector zones.

In particular, the previous studies indicated that the calculation biases for the most peripheral nodes (which at the same time are most important for the ex-core dosimetry calculations, even though they have relatively low power) can reach $\sim 20 \%$ within $\pm 1 \sigma$. That means that the uncertainties at the pin-wise level can reach even larger values. Thus, there is obviously a room for improvement of the neutron source specifications for the MCNP models, and one action in this direction would be to replace SIMULATE-3 code by SIMULATE5 which should provide more detailed and potentially more accurate data for neutron source specifications. It can be mentioned in passing that the impact of ND uncertainties on the power distribution at the peripheral fuel assemblies was analysed in work [2] and it was found within only $2 \%$.

In overall, there is probably some potential to combine the in-core validation studies and the ex-core reactor dosimetry validation cases under a common data assimilation framework, for improvement of both types of simulations. Such work can be considered for future activities at LRT/PSI.

\section{Acknowledgment}

This work is partially based on the results obtained by former PSI/EPFL \& PSI/ETHZ Master and PhD Students A. Dupré, R. Savelli and T. Zhu. This work was partially funded by swissnuclear through the PSI/SN R\&D project \# LRT-01 (2018-2019).

\section{References}

[1] A. Vasiliev, H. Ferroukhi, M.A. Zimmermann, R. Chawla, Ann. Nucl. Energy 34, 615-627 (2007).

[2] O. Leray, H. Ferroukhi, M. Hursin, A. Vasiliev, D. Rochman, Ann. Nucl. Energy 110, 547-559 (2017).

[3] A. Vasiliev, W. Wieselquist, H. Ferroukhi, S. Canepa, J. Heldt, G. Ledergerber, Proc. Int. Conf. MESC-2011 (2011).

[4] A. Vasiliev, W. Wieselquist, H. Ferroukhi, S. Canepa, J. Heldt, G. Ledergerber, Progress in Nucl. Sci. and Technol. 4, 99-103 (2014).

[5] A. Dupré, A. Vasiliev, H. Ferroukhi, A. Pautz, Ann. Nucl. Energy 85, 820-829 (2015).

[6] R. Savelli, A. Vasiliev, H. Ferroukhi, A. Pautz, Proc. ANS 2016 Student Conference (2016).

[7] M.B. Chadwick, et. al., Nucl. Data Sheets 107, 29313060 (2006).

[8] W. Wieselquist, T. Zhu, A. Vasiliev, H. Ferroukhi, Sci. Technol. Nucl. Ins. 2013, Article ID 549793, 1-15 (2013).

[9] T. Zhu, A. Vasiliev, H. Ferroukhi, A. Pautz, Ann. Nucl. Energy 75, 713-722 (2015).

[10] A. Vasiliev, D. Rochman, M. Pecchia, H. Ferroukhi, Energies 2016, 9 1039, 1-18 (2016).

[11] A. Dupre, A. Vasiliev, H. Ferroukhi, A. Pautz, Proc. ANS 2016 Student Conference (2014).

[12] A. Dupre. Master thesis public report, EPFL (2014).

[13] R. Savelli, Master thesis report, ETHZ (2016).

[14] I. Clifford, M. Pecchia, R. Puragliesi, A. Vasiliev, H. Ferroukhi, Nucl. Eng. Des. 330, 117-128 (2018).

[15] M. Pecchia, A. Vasiliev, H. Ferroukhi, A. Pautz, Ann. Nucl. Energy 105, 121-132 (2017).

[16] W. Wieselquist, H. Ferroukhi, K. Bernatowicz, Proc. Int. Conf. MEGC-2011 (2011). 